\title{
Using Hawksley Random Zero Sphygmomanometer as a Gold Standard May Result in Misleading Conclusions*
}

\author{
RÓNÁN M. CONROY, ' NEIL ATKINS, ${ }^{2}$ FÁINSÍA MEE, ${ }^{2}$ EOIN O'BRIEN ${ }^{2}$ and \\ KEVIN O'MALLEY ${ }^{2}$ \\ From the 'Department of Epidemiology \& Preventive Medicine, Royal College of Surgeons in Ireland, Dublin 2, and ${ }^{2}$ Blood Pressure Unit, \\ Beaumont Hospital, Dublin 7. Ireland
}

Conroy RM, Atkins N, Mee F, O'Brien E, O'Malley K. Using Hawksley random zero sphygmomanometer as a gold standard may result in misleading conclusions. Blood Pressure 1994; 3: 283-286.

We combined a database of paired blood pressure measurements taken using the Hawksley random-zero sphygmonanometer and a standard mercury sphygmomanometer and a database of paired measurements made on a SpaceLabs 90202 ambulatory blood pressure recorder and standard sphygmomanometer to determine how the SpaceLabs 90202 would have fared if it had been assessed against the Hawksley randomzero sphygmomanometer instead of a standard sphygmomanometer. The pooled database contained 255 triplicate readings. Using the standard sphygmomanometer as gold standard, the Spacelabs had a median error of $2 \mathrm{~mm} / \mathrm{Hg}$ for both systolic and diastolic. Against the Hawksley random-zero sphygmomanometer, median error was $-3 \mathrm{~mm}$ systolic and $-6 \mathrm{~mm}$ diastolic. The proportion of errors $>10 \mathrm{~mm}$ rose from $11 \%$ (systolic) and $9 \%$ (diastolic) with the standard sphygmomanometer to $16 \%$ and $29 \%$ with the Hawksley random-zero sphygmomanometer. Because it underestimates systolic and diastolic pressures, the use of the Hawksley random-zero sphygmomanometer as a gold standard may have resulted in misleading conclusions about performance of some automated BP recorders. Key words: hypertension, measurement, Hawksley random-zero sphygmomanometer.

\section{INTRODUCTION}

A controlled comparison of the Hawksley random zero sphygmomanometer and the standard mercury sphygmomanometer revealed a substantial lack of agreement between the two instruments [1]. Using the assessment protocol of the British Hypertension Society [2], the Hawksley under-read both systolic and diastolic pressures, by an average of $3.5 \mathrm{~mm}$ and $7.5 \mathrm{~mm} \mathrm{Hg}$, respectively. At the time of publication, we concluded that this finding might have serious implications for the interpretation of studies which had been carried out using the Hawksley instrument.

There are four main areas in which the Hawksley instrument had been used: the collection of population data, the evaluation of treatment trials, the selection of patients for studies - importantly, for studies of antihypertensive treatment-and the evaluation of automated blood pressure recording devices. It is the last application that this study addresses: what are the consequences of using a Hawksley rather than a mercury sphygmomanometer in evaluating an automated blood pressure recorder?

\section{MATERIALS AND METHODS}

Two sets of data were pooled to conduct the analysis.

*A copy of the data set upon which this report is based is available from the authors.
The first database was gathered during the evaluation of a Spacelabs 90202 automated blood pressure measuring instrument against a mercury sphygmomanometer [3]. It consisted of paired measurements, one taken with each instrument. The 85 subjects in this study were aged between 22 and 79 years, and were recruited from patients attending a blood pressure clinic, other hospital inpatients, and healthy hospital staff. This selection of subjects allowed the inclusion of a wide range of blood pressures. All subjects were in sinus rhythm and had an arm circumference of $<35 \mathrm{~cm}$.

The second database contained the data upon which the original report comparing the Hawksley and standard mercury instruments was based. Both sets of data were collected using the British Hypertension Society protocol [2] and described in detail in the original report [1]. A total of 258 readings were made using 86 subjects, aged 15-80 years. A pooled database was compiled from these two by adding matched Hawksley sphygmomanometer readings to the mercury/SpaceLabs database. For each systolic and diastolic reading made with the mercury sphygmomanometer in the mercury/SpaceLabs database, a mercury sphygmomanometer reading within 1 millimetre of mercury was identified in the mercury/ Hawksley database, and the corresponding Hawksley reading added to the mercury/SpaceLabs database. Where readings could not be matched exactly, they 
Table I. Evaluation of the SpaceLabs 90202 blood pressure recorder against a mercury sphygmomanometer and against a Hawksley random zero sphygmomanometer

\begin{tabular}{|c|c|c|c|c|}
\hline & \multicolumn{2}{|c|}{ Mercury (mm) } & \multicolumn{2}{|c|}{ Hawksley (mm) } \\
\hline & SBP & DBP & SBP & DBP \\
\hline $\begin{array}{l}\text { Mean error } \\
\text { Standard deviation } \\
\text { 25th/75th percentiles }\end{array}$ & $\begin{array}{l}1.9 \\
6.2 \\
-2 \text { to }+6\end{array}$ & $\begin{array}{l}1.9 \\
5.6 \\
-1 \text { to }+5\end{array}$ & $\begin{array}{l}-3.0 \\
7.6 \\
-8 \text { to }+2\end{array}$ & $\begin{array}{l}-6.2 \\
7.1 \\
-11 \text { to }-2\end{array}$ \\
\hline $\begin{array}{l}\text { Percent of errors in the interval: } \\
<5 \mathrm{~mm} \\
<10 \mathrm{~mm} \\
<15 \mathrm{~mm} \\
\text { over } 15 \mathrm{~mm}\end{array}$ & $\begin{array}{r}57.3 \% \\
83.9 \% \\
97.6 \% \\
2.3 \%\end{array}$ & $\begin{array}{r}64.7 \% \\
89.0 \% \\
97.3 \% \\
2.7 \%\end{array}$ & $\begin{array}{r}43.3 \% \\
78.3 \% \\
92.1 \% \\
7.9 \%\end{array}$ & $\begin{array}{l}35.0 \% \\
69.6 \% \\
10.2 \% \\
10.2 \%\end{array}$ \\
\hline BHS Grade* & $\mathrm{C}$ & B & D & D \\
\hline $\begin{array}{l}\text { Number of paired measurements } \\
\text { used in comparison }\end{array}$ & 255 & 255 & 254 & 246 \\
\hline
\end{tabular}

* BHS Grade: A, when cumulative $\%$ of readings $<5,<10$ and $<15 \mathrm{~mm} / \mathrm{Hg}$ are 80,90 and 95 respectively; B when 65,85 and 95; $C$ when 45,75 and 90 ; and $D$ when worse than $C$.

were matched to the first reading $1 \mathrm{~mm}$ above or below, and $1 \mathrm{~mm}$ added or subtracted from the corresponding Hawksley reading to adjust. (The decision as to whether to match upward or downward was based on the observation number: oddnumbered observations were downward-matched, even ones upward). Readings which could not be matched to within a millimetre were excluded from the study.

The resulting database contained triplicate readings: a reading made on the SpaceLabs 90202 , one made simultaneously on a mercury sphygmomanometer, and one made with a Hawksley sphygmomanometer when a mercury sphygmomanometer, read simultaneously, showed the same pressure as that recorded by the mercury sphygmomanometer in the SpaceLabs validation study. The use of random matching from an experimentally-derived dataset preserves the empirical pattern of discrepancy between the Hawksley and mercury instruments.

\section{RESULTS}

Table I shows the performance of the SpaceLabs 90202 assessed against the mercury sphygmomanometer and the Hawksley. There were 255 paired readings available upon which to base the comparison of the mercury and SpaceLabs instruments, and 254 systolic and 246 diastolic paired Hawksley/SpaceLabs readings. The loss of ten readings was because no matching mercury reading existed within $1 \mathrm{~mm}$ of the reference reading. The readings which could not be paired were mainly in the lower end of the blood pressure rangeof the ten, six had systolic pressures in the range
90-101 and diastolic pressures between 49 and $61 \mathrm{mmHg}$. One unmatched reading was of $190 /$ $143 \mathrm{mmHg}$.

The SpaceLabs instrument, as previously reported [3], had a slight tendency to underestimate both systolic and diastolic blood pressures. Its mean error (mercury minus SpaceLabs) was about $+2 \mathrm{mmHg}$ for both readings. The mean error changed direction and rose in size to $-3 \mathrm{mmHg}$ systolic and $-6.2 \mathrm{mmHg}$ diastolic when the Hawksley was substituted as a reference instrument. Using the standard sphygmomanometer, $57 \%$ of systolic errors, and $65 \%$ of diastolic errors were of less than $5 \mathrm{mmHg}$. When the Hawksley was used as a reference instrument, this fell to $43 \%$ and $35 \%$. Likewise, the percentage of errors of more than $15 \mathrm{mmHg}$ rose from $2 \%$ (systolic) and $3 \%$ (diastolic), when the mercury instrument was used as a reference, to $8 \%$ (systolic) and $10 \%$ (diastolic) when the Hawksley was substituted. The two reference instruments differed significantly in the distribution of errors in each category- $\chi^{2}=10.3, \mathrm{df}=3, p=$ 0.016 for systolic pressure and $\chi^{2}=55.3, \mathrm{df}=3$, $p<0.001$ for diastolic pressure. The net effect of changing from the mercury to the Hawksley as a reference instrument is a negative shift in the error distribution.

The British Hypertension Society grading system was used to rate the performance of the SpaceLabs 90202. When rated against the mercury sphygmomanometer, it achieved a rating of $\mathrm{B}$ for diastolic accuracy and $\mathrm{C}$ for systolic. This dropped to a D grade in both cases when the Hawksley was used as a reference instrument.

We also examined the relationship between the 


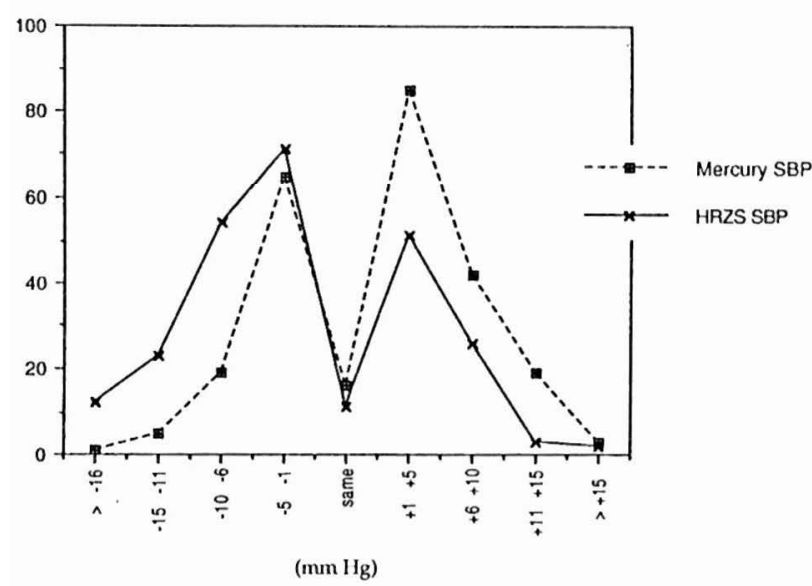

Fig. 1. Systolic pressure errors comparing SpaceLabs 90202 with HRZS or mercury sphygmomanometer.

size of the difference between the SpaceLabs machine and reference instrument as a function of the blood pressure, as measured by the reference instrument. When the mercury sphygmomanometer was used as a reference instrument, systolic errors increased by an average of $0.8 \mathrm{mmHg}$ with every $10 \mathrm{~mm}$ increase in systolic pressure, and diastolic errors by $0.7 \mathrm{mmHg}$. With the Hawksley as reference instrument, systolic pressure errors increased by 0.8 for a $10-\mathrm{mm}$ increase in pressure and diastolic pressure errors by $1.4 \mathrm{mmHg}$.

\section{DISCUSSION}

In a recent review of the consequences of the inaccuracy of the Hawksley sphygmomanometer, we speculated that the use of the instrument as a reference in the validation of automated blood pressure recording devices might result in a misleadingly unfavourable evaluation [4]. The present study design, by pooling data from two studies, each of which used a Hawksley sphygmomanometer in comparison with another instrument, simulates an experiment in which triplicate blood pressure readings are taken simultaneously using a mercury sphygmomanometer, a Hawksley Random-zero sphygmomanometer and a Spacelabs 90202 automated blood pressure recorder. The technique used, that of pooling two databases based on random matching, has the advantage that it incorporates no prior assumptions about the pattern of error of any of the instruments being compared, which would have been the case, for example, if we had substituted for each mercury reading the mean of all Hawksley readings taken at that pressure.

As reported previously, the SpaceLabs 90202 blood pressure recording instrument has a reasonable level of

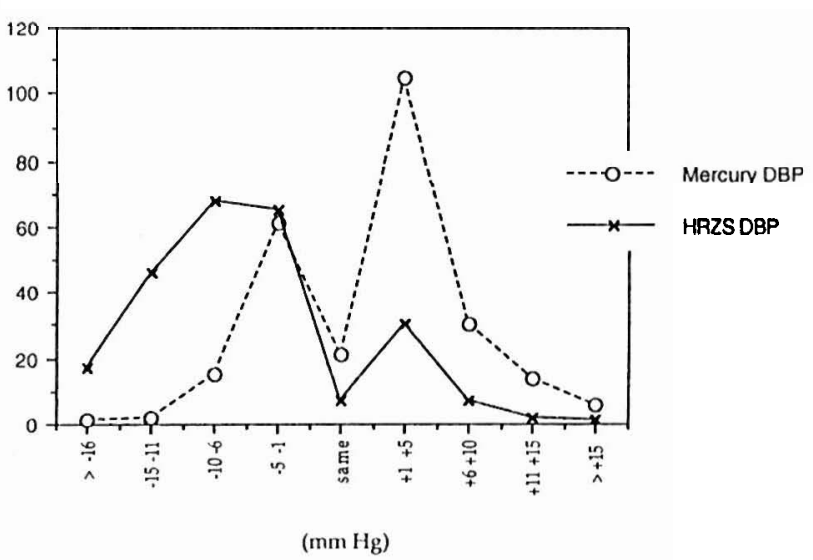

Fig. 2. Diastolic pressure errors comparing SpaceLabs 90202 with HRZS or mercury sphygmomanometer.

agreement with a standard sphygmomanometer. The effect of replacing the standard instrument with a Hawksley sphygmomanometer was to reverse the direction of the average measurement error found, and to demote the machine from BHS grades $\mathrm{C}$ and $\mathrm{B}$, for systolic and diastolic accuracy respectively, to grade $\mathrm{D}$ overall - the lowest rating of accuracy in the BHS grading system. Such a report, had it been published, might have been commercially damaging. Furthermore, the manufacturers might have been impelled to recalibrate their instrument so that it agreed as closely as possible with the Hawksley, thereby enshrining the measurement errors of the latter instrument in their own. This would have been ironic, because the SpaceLabs instrument, according to the data presented, is more accurate an instrument than the Hawksley.

The results presented highlight the consequences of the continuing use of the Hawksley. We have reviewed these extensively in a recent publication [4], and we can here only reiterate our recommendations that any blood pressure measuring instruments which have been assessed only against the Hawksley instrument should be reassessed against a mercury sphygmomanometer, and that the use of the Hawksley as a reference instrument for such assessments be discontinued.

\section{ACKNOWLEDGEMENTS}

We acknowledge with gratitude support from the Charitable Infirmary Charitable Trust, the Royal College of Surgeons in Ireland and Beaumont Hospital.

\section{REFERENCES}

1. O'Brien E, Mee F, Atkins N, O'Malley K. Inaccuracy of 
the Hawksley random zero sphygmomanometer. Lancet 1990; 336: 1465-8.

2. O'Brien E, Petrie J, Littler W, de Swiet M, Padfield PL, O'Malley K, Altman D, Bland M, Atkins N. The British Hypertension Society Protocol for the evaluation of automated and semi-automated blood pressure measuring devices with special reference to ambulatory systems. J Hypertens 1990; 8: 607-19.

3. O'Brien E, Mee F, Atkins N, O'Malley K. Evaluation of the SpaceLabs 90202 non-invasive ambulatory recorder according to the AAMI standard and BHS criteria. J Hum Hypertens 1991; 5: 223-6.

4. Conroy RM, O’Brien E, O'Malley K, Atkins N.
Measurement error in the Hawksley random zero sphygmomanometer: what damage has been done and what can we learn? BMJ 1993; 306: 1319-22.Submitted April 26, 1993; accepted July 7, 1993

Address for correspondence:

Rónán Conroy

Department of Epidemiology

Royal College of Surgeons

Mercer Building

Lower Mercer Street

Dublin 2

Ireland 